\title{
Potential Lethal Co-infections in COVID-19: A Study Based on Literature Review
}

\author{
Shiv Nandan Sah ${ }^{1 *}$, Arjun Ghimire ${ }^{1}$, Ranjit Kumar $\mathrm{Sah}^{2}$, Pradeep Kumar $\mathrm{Sah}^{3}$, Neena \\ Capalash $^{4}$, and Prince Sharma ${ }^{5}$ \\ ${ }^{1}$ Department of Microbiology, Central Campus of Technology, Tribhuvan University, Dharan, Nepal. \\ ${ }^{2}$ Department of Microbiology, Ramswaroop Ramsagar Multiple Campus, Tribhuvan University, Janakpurdham, \\ Dhanusha, Nepal. ${ }^{3}$ Department of Microbiology, Tri-Chandra Multiple Campus \\ Tribhuvan University, Ghantaghar, Kathmandu, Nepal. ${ }^{4}$ Department of Biotechnology, Panjab \\ University, Chandigarh, India. ${ }^{5}$ Department of Microbiology, Panjab University, Chandigarh, \\ India.
}

\section{*CORRESPONDENCE:}

Shiv Nandan Sah

Department of Microbiology, Central

Campus of Technology, Tribhuvan

University, Dharan, Nepal.

Email: sahshivnandan96@gmail.com

ISSN : 2382-5359(Online), 1994-1412(Print)

DIO:

https://doi.org/10.3126/njst.v20i1.39448

\section{ACCESS THE ARTICLE ONLINE}

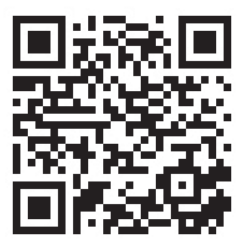

\section{CONFILICT OF INTEREST: None}

Copyright: The Author(s) 2019. This is an open access article under the $\underline{\text { CC BY license. }}$

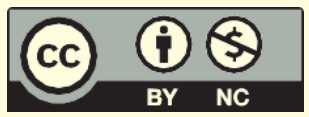

\begin{abstract}
Co-infection with other respiratory pathogens has been reported in patients with COVID-19. Common respiratory pathogens can infect as co-pathogens during SARS-CoV-2 infections. The aim of this article is to spread knowledge regarding possible co-infections during COVID-19, and reduce their occurrence. Google scholar was used to search the literature for possible co-infections in the people with COVID-19 and reviewed the existing published data. In most cases, co-infections are common due to Streptococcus pneumoniae, Staphylococcus aureus, Klebsiella pneumoniae, Mycoplasma pneumoniae, Chlamydia pneumonia, Legionella pneumophila, and Acinetobacter baumannii. Prevalence of fungal and viral coinfections is low. However, Candida species and Aspergillus flavus arethe common co-infective fungi. Viruses such as Influenza, Corona virus, Rhinovirus/ Enterovirus, Parainfluenza, Metapneumo virus, Influenza B virus, and Human immunodeficiency virus have also been reported as co-infecting agents during COVID-19. Influenza A was one of the most common co-infective viruses, which may have caused initial false-negative results of a real-time RT-PCR for severe acute respiratory syndrome corona virus 2 (SARS-CoV-2). The prevalence of co-infections could be up to 50\% among non-survivors. Only newly developed syndromic multiplex panels that incorporate SARSCoV-2 may facilitate the early detection of co-infections. The suitable antimicrobial agents can be recommended for the co-infections caused by other respiratory pathogens during COVID-19.
\end{abstract}

Keywords: Respiratory pathogens, RT-PCR, SARS-CoV-2, syndromic multiplex panels 


\section{INTRODUCTION}

In December 2019, a novel corona virus (nCoV) was mounted in Wuhan, Huanan, province of Hubei, and has become sizeable international precedence due to the outbreak of pneumonia, where farm animals become exchanged (traded) (Zaki et al. 2012). Severe acute respiratory syndrome corona virus 2 (SARS-CoV-2) primarily affects the respiratory system, though the involvement of other organs, such as heart, kidney, and bowel, has also been observed (Chen et al., 2020). The unconventional new virus SARS-CoV-2 is the seventh recognized $\mathrm{CoV}$ to infect human beings from this viral circle of relatives. In the beginning, on December 12 2019, an unexplained case of pneumonia became identified in Wuhan. Laboratory exams eliminated suspected influenza and different CoVs (Awami et al. 2020). On January 7 2020, China's authorities declared the new CoV type (Imai et al. 2020). On January 12 2019, nCoV was designated by WHO, and on February 11 2020, It was named as COVID-19. A total of 2,355,853 recorded cases were registered, with 164,656 fatalities as of the April 202020 (Din \& Bopanna 2020). The recent outbreak of the novel corona virus called severe acute respiratory syndrome-coronavirus-2 (SARS-COV-2) was declared a pandemic on March 112020 by the WHO (WHO 2020). The current primary modality of testing is a real-time reverse transcription-polymerase chain reaction (RT-PCR) (Corman et al. 2020; Loeffelholz \& Tang 2020). The virus that causes COVID-19 infects humans of every age. The danger of intense disease progressively increases with age beginning from around 40 years. Adults on this age variety must guard themselves and shield others that can be more inclined (WHO 2020). These are older people (over 60 years old); and those with underlying medical conditions (such as cardiovascular disease, diabetes, chronic respiratory disease, and cancer (Koinyeneh 2020).

The outbreak of COVID-19 pandemic led to major health problems for the global population. The virus has been found to be transmitted through aerosols released from coughing and sneezing (Dhand \& Li 2020). Hence, human-to-human transmission of this virus was confirmed through the dissemination of respiratory droplets or aerosols. Accordingly, Wu, (2020) reported that the disease is rapidly transmitted by infected travelers to the other geographical regions and territories.

In most cases of death due to COVID-19, Co-infections with common respiratory pathogens has been reported in patients. Common respiratory pathogens can infect as copathogens during SARS-nCoV-2 infections.

\section{REVIEW AND DISCUSSION}

The recognition of SARS-CoV-2 infection is essential as it enables the implementation of appropriate infection control measures and possible promising antiviral therapy, but clinicians should not neglect the possibility of SARSCoV-2 co-infection (Lai et al. 2020).

Respiratory viral infections predispose patients to coinfections leading to increased disease severity and mortality (Cox et al. 2020). Most fatalities in the 1918 influenza outbreak were due to subsequent bacterial infection, particularly with Streptococcus pneumoniae (Morens et al. 2008).

Poor outcomes in the 2009 H1N1 influenza pandemic were also associated with bacterial co-infections, although few studies captured these data (MacIntyre et al. 2018). Despite the validated significance of co-infections within the severity of respiration illnesses, they are understudied at some stage in massive outbreaks of respiratory infections. Within the cutting-edge state of affairs of corona virus disease 2019 (COVID-19) pandemic, 50\% of sufferers with COVID-19, who've died, had secondary bacterial infections (Zhou et al. 2020). (Chen et al. 2020) have recorded both bacterial and fungal co-infections. Although $71 \%$ of the admitted patients with COVID-19 received antibiotic drugs, no information is available on the antimicrobial sensitivities of the organisms identified or on the type and duration of antimicrobial treatment. Chronic obstructive pulmonary ailment (COPD) is a threat component for extreme COVID-19 sickness, and lots of sufferers with COPD can have underlying persistent bacterial infections before extreme acute respiratory syndrome Corona virus2 (SARS-CoV-2) infections, but this critical record is not being pronounced. More data on co-infections are urgently required to set up their significance in COVID-19 severity and mortality (Chen et al. 2020).

Diagnosing co-infections is complex. The organism might be carried by the patient before the viral infection and might be part of an underlying chronic infection, or picked up nosocomial. In the UK, National Institute for Health and Care Excellence (NICE), treatment guidance for severe acquired pneumonia is broad-spectrum antibiotic coamoxiclav plus a macrolide to cover atypical organisms. Currently, antibiotic use is high (74.5\%) among patients with COVID-19 who are admitted to intensive care units, rendering culture-based microbiological testing less sensitive. Patients with COVID-19 are kept on invasive mechanical ventilation for a long time (mean 9.1 days [SD 5.5]), increasing chances of hospital and ventilator 
acquired infections. Hence, early diagnosis of co-infection is required, preferably using methods capable of detecting a broad range of potential pathogens and antimicrobial resistance, with subsequent monitoring for infection development (Cox et al. 2020). Hence, for diagnosis of Co-infections in COVID-19, sufferers must be admitted in intensive care units (ICUs) and sampled longitudinally at some point of the ailment direction by using the lifestyle-unbiased techniques inclusive of whole-genome metagenomics which can figure out complicated blended infections without previous goal choice (Charalampous et al. 2019).

A large proportion of COVID-19 patients with a coinfection of seasonal respiratory pathogens in Qingdao, northeast China, differed significantly from Wuhan's patients, central China. Precautions are needed when dealing with COVID-19 patients beyond the epidemic centre who have co-infection with other respiratory pathogens (Xing et al. 2020). It was found that a low frequency of microbiologically confirmed bacterial coinfection was present in confirmed SARS-CoV-2 patients admitted to secondary care in an urban UK setting (Huges et al. 2020).

Based on previous studies on severe corona virus infections, serological evidence among SARS patients indicated incidences of acute or recent Chlamydophila pneumonia (30\%) or Mycoplasma pneumoniae (9\%) infections respectively (Zahariadis et al. 2006). Six pieces of the research reported the incidence of bacterial co-contamination, and M. pneumoniae, Legionella pneumophila, Streptococcus pneumoniae, and $C$. pneumoniae have been identified as co-pathogens. Eight studies stated viral co-infections; Rhinovirus/ Enterovirus and Influenza A have been the commonest co-pathogen, Corona virus, breathing syncytial virus, Parainfluenza, Metapneumovirus, and Influenza B virus had also been stated as co-pathogens (Chen et al. 2020).

The other co-pathogens included HIV, $M$. pneumoniae, Staphylococcus aureus, $L$. pneumophila, Klebsiella pneumoniae, Acinetobacter baumannii, Aspergillus flavus (Lai et al. 2020). COVID19 co-infection with other common respiratory pathogens such as Mycoplasma pneumonia may exacerbate clinical symptoms, increase morbidity, and cause prolonged ICU stay if left undetected or untreated. Clinicians managing patients with COVID-19 infection should be mindful of coinfections with common respiratory pathogens during this COVID-19 outbreak and screen for these with appropriate microbiologic tests (Fan et al. 2020).
Among 35 patients presenting lung SARS-CoV-2 infection and concomitant positive bronchial aspirates, 28 (80\%) were colonized by either fungi or $P$. aeruginosa. Fungi are the predominant causes of morbidity and mortality in immune compromised subjects, even as $P$. aeruginosa is the maximum commonplace gram-terrible pathogen causing pneumonia associated with worse clinical consequences (Jiang 2016; Curran et al. 2020).

On the other hand, in non-COVID-19 patients admitted to the ICU in 2019 , only $20 \%$ of first bronchial aspirate cultures presented fungi (C. albicans, C. glabrata, $C$. tropicalis, C. parapsilosis, \& A. fumigatus) or $P$. aeruginosa colonization (Rossato 2020). The findings of co-infection with exceptional respiration pathogens and SARS-CoV-2 undertaking using an algorithm requiring prior trying out for viral breathing and bacterial pathogens earlier than checking out for SARS-CoV-2 (Khaddour et al. 2020).

Various studies would provide valuable surveillance data on the pathogens causing co-infections and antimicrobial resistance in the intensive care setting, thereby informing antibiotic prescribing policy. Rapid characterization ofcoinfection is essential in managing and treating of the most severe COVID-19 cases, could help save lives, and improve antimicrobial stewardship throughout the pandemic (Cox et al. 2020).

Co-infection has been reported in patients with severe acute respiratory syndrome (SARS) and the Middle East respiratory syndrome, but there is limited knowledge on co-infection among patients with corona virus disease 2019 (COVID-19). The prevalence of co-infection was variable among COVID-19 patients in different studies; however, it could be up to $50 \%$ among non-survivors (Lai et al. 2020). Co-pathogens covered microorganism, together with Streptococcus pneumoniae, Staphylococcus aureus, Klebsiella pneumoniae, Mycoplasma pneumoniae, Chlamydia pneumonia, Legionella pneumophila, and Acinetobacter baumannii; Candida species and Aspergillus flavus; and viruses together with Influenza, Coronavirus, Rhinovirus/Enterovirus, Parainfluenza, Metapneumovirus, Influenza B virus, and Human Immunodeficiency virus. Influenza A turned into one of the maximum common coinfective viruses, which might also have induced initial false-poor results of a real-time reverse-transcriptase polymerase chain reaction for intense acute respiratory syndrome corona virus 2 (SARS-CoV-2) (Lai et al. 2020)

Many approved multiplex PCR panels are available for the early diagnosis of possible non-SARS-CoV-2 respiratory pathogens in COVID-19 patients(Lee et al., 2019; Lai 
et al., 2020). Fortunately, SARS-CoV-2 has been rapidly incorporated into preexisting syndromic multiplex panels, such as QIAstat-Dx® Respiratory 2019-nCoV Panel (Qiagen, the Netherlands) and BioFire FilmArray RP2.1 (BioFire FilmArray Respiratory Panel-2 plus SARSCoV-2; bioMerieux, France). In addition to SARS-CoV-2, many other common respiratory pathogens, including bacteria and viruses, can be simultaneously identified using the QIAstat-Dx® Respiratory 2019-nCoV Panel. Using this SARS-CoV-2 containing syndromic/co-infection test, the risk of under-diagnosis of co-infection can be primarily reduced during the COVID-19 pandemic (Lai et al. 2020).

\section{FUTURE PERSPECTIVE}

Among COVID-19 patients, co-infection with other respiratory pathogens is possible. Further large-sample, well-designed studies should be carried out to investigate the prevalence of COVID-19 co-infection, risk of coinfection, microbiological distribution, and impact of coinfection on the clinical outcomes of COVID-19 patients. Based on more data obtained regarding SARS-CoV-2 with co-infection, suitable antimicrobial agents in suspected COVID-19 cases can be recommended. Awareness among people about all the possible infectious agents and their routes of infections should be raised to prevent coinfections during COVID-19.

\section{ACKNOWLEDGEMENT}

The authors would like to thank the Department of Microbiology, Central Campus of Technology, Dharan, Tribhuvan University, Nepal, for providing library and internet facilities to complete this review work.

\section{REFERENCES}

1. Amawi, H., G.I.A. Deiab, A.A.A. Aljabali, K. Dua and M.M. Tambuwala. 2020. COVID-19 pandemic: an overview of epidemiology, pathogenesis, diagnostics and potential vaccines and therapeutics. Therapeutic Delivery 11(4): 245-268

2. Charalampous, T., G.L. Kay, H. Richardson, A. Aydin, R. Baldan, C. Jeanes, D. Rae, S. Grundy, D.J. Turner, J. Wain, R.M. Leggett, D.M. Livermore and J. O'Grady. 2019. Nanopore metagenomics enables rapid clinical diagnosis of bacterial lower respiratory infection. Nature Biotechnology 37: 783-792.

3. Chen, N., M. Zhou, X. Dong, J. Qu, F. Gong, Y. Han, Y. Qui, J. Wang, Y. Liu, Y. Wei, J. Xia, T. Yu, X. Zhang and L. Zhang. 2020. Epidemiological and clinical characteristics of 99 cases of 2019 novel coronavirus pneumonia in Wuhan, China: a descriptive study. The Lancet 395(10223): 507-513.

4. Corman,V.M., O. Landt, M. Kaiser, R. Molenkamp, A. Meijer, D. KW. Chu, T. Bleicker, S. Brünink, J. Schneider, M.L. Schmidt, D. GJC. Mulders, B.L. Haagmans, B.V. Veer, S.V. Brink, L. Wijsman, G. Goderski, J.-L. Romette, J. Ellis, M. Zambon, M. Peiris, H. Goossens , C. Reusken , M.PG. Koopmans and C. Drosten. 2020. Detection of 2019 novel coronavirus (2019-nCoV) by real-time RT-PCR. Eurosurveillance 25(3): 2000045.

5. Cox M.J., N. Loman, D. Bogaert and J. O'Grady. 2020. Co-infections: potentially lethal and unexplored in COVID-19. The Lancet Microbe 1(1): e11.

6. Curran, C.S., T. Bolig and P. Torabi-Parizi. 2020. Mechanisms and targeted therapies for Pseudomonas aeruginosa lung infection. American journal of respiratory and critical care medicine 197(6): 708727.

7. Dhand, R. and J. Li. 2020. Coughs and Sneezes: Their Role in Transmission of Respiratory Viral Infections, Including SARS-CoV-2. American Journal of Respiratory and Critical Care Medicine 202(5): 651-659.

8. Din, M.A.U. and L.K.T. Boppana. 2020. An update on the 2019-nCoV outbreak. American Journal of Infection Control 48(6): 713.

9. Fan, B.E., K.G.E. Lim, V.C.L. Chong, S.S.W. Chan, K.H. Ong and P. Kuperan. 2020. COVID-19 and Mycoplasma pneumoniae co-infection. American Journal of Hematology 95(6):723-724.

10. Hughes, S., O. Troise, H. Donaldson, N. Mughal and L.S.P. Moore. 2020. Bacterial and fungal co-infection among hospitalized patients with COVID-19: a retrospective cohort study in a UK secondary-care setting. Clinical Microbiology and Infection 26(10): 1395-1399.

11. Imai, N., I. Dorigatti, A. Cori, C. Donnelly, S. Riley and N. M. Ferguson. 2020. Report 2: Estimating the potential total number of novel Corona virus cases in Wuhan City, China. Imperial College London: 1-7.

12. Jiang, S. 2016. Immunity against fungal infections. Immunology and Immunogenetics Insights 8: III. S38707.

13. Khaddour, K., A. Sikora, N. Tahir, D. Nepomuceno and T. Huang. 2020. Case report: the importance of novel 
corona virus disease (COVID-19) and co infection with other respiratory pathogens in the current pandemic. The American Journal of Tropical Medicine and Hygiene 102(6): 1208-1209.

14. Koinyeneh, G.C. 2020. Liberia: Denial, Mistrust, Recriminations Mar COVID-19 Fight in Nimba County Amid Surge in Cases. Front Page Africa. https://allafrica.com/stories/202005040778.html.

15. Lai, C.-C., C.-Y. Wang and P-R Hsueh.2020. Coinfections among patients with COVID-19: The need for combination therapy with non-anti-SARS-CoV-2 agents? Journal of Microbiology, Immunology and Infection 53: 505-512.

16. Lee, S.H., S.-Y. Ruan, S.-C. Pan, T.-F. Lee and J.Y. Chien. 2019. Performance of a multiplex PCR pneumonia panel for the identification of respiratory pathogens and the main determinants of resistance from the lower respiratory tract specimens of adult patients in intensive care units. Journal of Microbiology, Immunology and Infection 52(6): 920-928.

17. Loeffelholz, M. J. and Y.-W. Tang. 2020. Laboratory diagnosis of emerging human corona virus infectionsthe state of the art. Emerging microbes \& infections 9(1): 747-756.

18. MacIntyre C.R., A.A. Chughtai, M. Barnes, I. Ridda, H. Seale, R. Toms and A. Heywood. 2018. The role of pneumonia and secondary bacterial infection in fatal and serious outcomes of pandemic influenza a(H1N1) pdm09. BMC Infectious Diseases 18: 637

19. Morens D.M., J.K. Taubenberger and A.S. Fauci. 2008. Predominant role of bacterial pneumonia as a cause of death in pandemic influenza: implications for pandemic influenza preparedness. The Journal of Infectious Diseases 198: 962-970.

20. Rossato, L., F.J. Negrão and S. Simionatto. 2020. Could the COVID-19 pandemic aggravate antimicrobial resistance? American Journal of Infection Control 48(9): 1129-1130.
21. WHO. 2020. Coronavirus disease 2019 (COVID19): situation report, 51. World Health Organization. https://apps.who.int/iris/handle/10665/331475

22. Wu, S.C. 2020. Progress and Concept for COVID-19 Vaccine Development. Biotechnology Journal 15(6): e2000147

23. Xing Q., G. Li, Y. Xing, T. Chen, W. Li, W. Ni, K. Deng, R. Gao, C. Chen, Y. Gao, Q. Li, G. Yu,J. Tong, W. Li, G. Hao, Y. Sun, A. Wu, Z. Li and S. Pan. 2020. Precautions are needed for COVID-19 Patients with Co-infection of Common Respiratory Pathogens. The Lancet preprint.

24. SSRN: https://ssrn.com/abstract=3550013 or http:// dx.doi.org/10.2139/ssrn.3550013

25. Zahariadis, G., T.A. Gooley, P. Ryall, C. Hutchinson, M.I. Latchford, M.A. Fearon, F.B. Jamieson, S. Richardson, T. Kuschak, and B. Mederski. 2006. Risk of Ruling out Severe Acute Respiratory Syndrome by Ruling in another Diagnosis: Variable Incidence of Atypical Bacteria Co infection Based on Diagnostic Assays. Canadian Respiratory Journal 13: 862797.

26. Zaki, A.M., S.V. Boheemen, T.M. Bestebroer and A.D.M.E. Osterhaus. 2012. Isolation of a novel corona virus from a man with pneumonia in Saudi Arabia. New England Journal of Medicine 367(19): 1814-1820.

27. Zhou, F., T. Yu, R. Du, G. Fan, Y. Liu, Z. Liu, J. Xiang, Y. Wang, B. Song, X. Gu, L. Guan, Y. Wei, H. Li, X. Wu, J. Xu, S. Tu, Y. Zhang, H. Chen, and B. Cao. 2020. Clinical course and risk factors for mortality of adult in patients with COVID-19 in Wuhan, China: a retrospective cohort study. The Lancet 395 (10229): 1054-1062. 